\title{
Pre-implementation Genetics for Aneuploidy Interpretation in PGT-A Goals Indications and Improve Infertility Outcomes, ART, and How to Perform PGT-A
}

\author{
Aya Al-ibraheemi ${ }^{1 *}$ Mustafa Zakaria $^{2 *}$, Noureddine Louanjli ${ }^{3}$, \\ Mohamed Zarqaoui ${ }^{4}$, Mohamed Ennaji ${ }^{5}$, Senhaji R Wassym ${ }^{6}$, Ritu S \\ Santwani ${ }^{7}$, Nisrine En-naciri ${ }^{8}$, Hafida Tarik $^{8}$ and Romaissa Boutiche ${ }^{9}$ \\ ${ }^{1}$ Embryologist, Graduated from University of Nottingham, United Kingdom \\ ${ }^{2}$ Reproductive Biology, and Assisted Reproductive Technology, Consultant at IRIFIV \\ Fertility Center, Administrative Deputy and Writer for the ART IRIFIV Scientific \\ Research Group (AISRG) Casablanca, Morocco \\ ${ }^{3}$ Head of LABOMAC Laboratory of Clinical Analysis and Assisted Reproductive \\ Technology IRIFIV Fertility Center, AFC Fertility Center - Executive Vice President of \\ the ART IRIFIV Scientific Research Group (AISRG) Casablanca, Morocco \\ ${ }^{4}$ Endoscopic Surgery Obstetrics and Gynecology FR, Coordinator of IRIFIV \\ Fertility Center, Head of the ART IRIFIV Scientific Research Group (AISRG) \\ Casablanca, Morocco \\ ${ }^{5}$ Senior Clinical Embryologist at IRIFIV Fertility Center, Member of the Scientific \\ Research Group and Researcher in the Scientific Research Group, Casablanca, \\ Morocco \\ ${ }^{6}$ Gynecologist and Obstetrician, Endoscopic Surgeon and Fertility Expert, A \\ Research in Art Irifiv Scientific Research Group Associated Practitioner at IRIFIV \\ Fertility Center - Casablanca, Morocco \\ ${ }^{7}$ Gynaecologist and Obstetrician, ART-Singapore, Honorary Professor, India \\ ${ }^{8}$ Senior Clinical Embryologist, Laboratory IVF Agadir, Souss-Massa, Member of the \\ Scientific Research Group and Researcher in the Scientific Research Group, Agadir, \\ Morocco \\ ${ }^{9}$ Senior Clinical Embryologist, Laboratory IVF Algeria, Rotaby Fertility Center \\ Algeria - Algiers, Member of the Scientific Research Group and Researcher in the \\ Scientific Research Group, Algeria \\ *Corresponding Author: Mustafa Zakaria, Consultant at IRIFIV Fertility Center, \\ Administrative Deputy and Writer for the ART IRIFIV Scientific Research Group \\ (AISRG), Casablanca, Morocco.
}

\author{
Received: November 21, 2020 \\ Published: December 15, 2020 \\ C All rights are reserved by Mustafa \\ Zakaria., et al.
}

\begin{abstract}
Preimplantation genetic testing for aneuploidy PGT-A has been widely applied today in assisted reproductive technology treatments centers over the world to select the health embryos for transfer with euploid cells and to enhance clinical outcomes such as embryo implantation, clinical pregnancy, and live birth rates. The PGT-A can be performed in during an IVF cycle, after fertilization where a biopsy can be taken from the blastocysts to be genetically tested. PGT-A indication can be to decrease recurrent miscarriage, increase the pregnancy rate in advance maternal age, and increase single embryo transfer. This review also demonstrates new PGT-A method which is called non-invasive PGT-A, to prevent the embryo development distracting and removing extra cells from the embryo, through analyzing leaked DNA.

Keywords: Infertility and ART; PGT and PGT-A; How to Perform PGT-A; Blastocytes Biopsy Steps; PGT-A Goals/Indication; PGT-A Interpretation; Non-invasive PGT-A
\end{abstract}




\section{Abbreviations}

IVF: In-vitro Fertilization; ICSI: Intracytoplasmic Sperm Injection; ART: Assisted Reproduction Technology; PGT-A: Preimplantation Genetic Testing For Aneuploidy; PGT-SR: Chromosomal Structural Rearrangements; PGT-M: Preimplantation Genetic Testing for Monogenic; FISH: Fluorescence In Situ Hybridization; PCR: Polymerase Chain Reaction; RIF: Recurrent Implantation Failure; AMA: Advanced Maternal Age; RM: Recurrent Miscarriage; SET: Single Embryo Transfer; KS: Klinefelter Syndrome; SMF: Severe Male Factor; TE: Trophectoderm; niPGT-A: Noninvasive Preimplantation Genetic Testing for Aneuploidy.

\section{Introduction}

Infertility and assisted reproductive technology

Infertility is a frequently common health condition that can be described as the failure of a couple to conceive a child after two years of unprotected sex. Unfortunately, infertility has grown increasingly due to environmental and genetic factors, such as obesity, smoking, alcohol and drug use, as well as environmental pollution (Lal., et al. 2020). Therefore, numbers of infertile individuals have quickly risen past $15 \%$ of reproductive-age couples [1]. Consequently, assisted reproduction technology (ART), and more particularly during vitro fertilization (IVF) have considered the burden of this increasing demand. These technologies are becoming an increasingly more common means to conceiving a child While IVF is an opportunity for several couples to conceive, rates of successful IVF, implementation and live birth flutter around 50\%, reducing in possibility with advancing maternal age and other medical difficulties (Fragouli., et al. 2018) [2]. Normally, embryos are picked for transfer based on morphology grading. Patient embryos usually undergo biopsy and consequent chromosomal analysis, in the case of recurring miscarriages or advanced maternal age. This procedure is referred to as Preimplantation genetic testing for aneuploidy (PGT- A) (Lal., et al. 2020).

\section{PGT and PGT-A}

Generally, PGT is described as a procedure made to examine and analyse the DNA from oocytes (polar bodies) or embryos (cleavage stage or blastocyst) for HLA typing or to define genetic abnormalities. PGT can include PGT for aneuploidy (PGT-A), PGT for monogenic/single gene defects (PGT-M) and PGT for chromosomal structural rearrangements (PGT-SR) (Kokkali., et al. 2020) [3]. Aneuploidy is the most frequent genetic irregularity found in humans, and it's considered to be a common cause of a failed implantation, pregnancy loss, and congenital disabilities. Usually, diploid cells carry 46 chromosomes, which is known as euploidy cells. Aneuploidy is a remodelled condition including a deviation in copy number from multiples of 23. A typical example is a trisomy condition, resulting in 47 chromosomes. Aneuploidy can change various chromosomes in a cell, in some cases this condition is referred to as complex aneuploids, or result in nullisomy or polysomy, where none or multiple copies of individual chromosomes are present figure 1 spell check and correct (Viotti M, 2020).

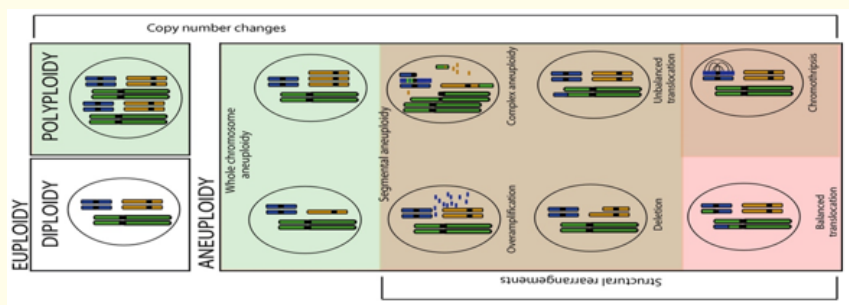

Figure 1: The figure above illustrate the difference between euploidy and aneuploidy.

In addition to the different types of aneuploidy. Cells with complex aneuploid karyotypes contain both chromosome copy number changes and structural rearrangements (Dürrbaum and Storchová, 2015).

PGT started as an experimental test in the 1990s using polymerase chain reaction (PCR)-based techniques applied firstly for sex selection and the detection of monogenic disorders. A few years later, Interphase fluorescence in situ hybridization (FISH) was introduced and became the standard technique for embryos sexing and for identifying numerical and structural chromosomal irregularities (Kokkali., et al. 2020) [4]. However, PGT-A is usually carried out for in vitro fertilization (IVF) patients, aiming to increase pregnancy rates per embryo transfer and reducing miscarriage rates. Another benefit of PGT-A is to increase elective single embryo transfer and decreased time to pregnancy. PGT-A milestones also include it application for advanced maternal age (AMA), recurrent implantation failure (RIF) and severe male factor (SMF) and couples with normal karyotypes who have experienced recurrent miscarriage (RM) (Harper., et al. 2018).

\section{How to perform PGT-A}

PGT-A can only be performed during an IVF cycle, thus after egg retrieval, IVF or ICSI is performed to fertilize the egg; following fertilization the fertilized eggs become blastocysts after 5-6 days. Based on morphology, blastocysts with good morphology are biopsied on days 5-6, then they are frozen to be transferred later to the mother uterus if they pass the PGT-A tests figure 2.

There are three main types of embryo biopsy, which include polar body biopsy, cleavage stage biopsy and blastocyst biopsy. Blastocyst biopsy is an emerging technique, which provides more cell to analysis since it contains the inner cell mass with the embryo (ectoderm, mesoderm and endoderm), vitelline and vesicle am- 
nion; in addition to the trophectoderm with non-embryonic tissues (chorion, placenta, umbilical cord). Blastocytes biopsy has several advantages such as it can be beneficial for monogenic diseases, a lower degree of mosaicism, and it keeps the ICM fully intact. However, blastocytes biopsy limitation sets in post-zygotic mosaicism (anucleate, multinucleation), embryo arrest and time for diagnosis (Boada and Veiga, 2010) [2].
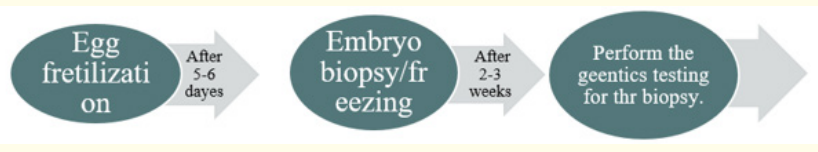

Figure 2: The figure above illustrate the main steps of performing PGT-A.

\section{Blastocytes biopsy steps}

- Zona Pellucida drilling, using Laser or mechanical biopsy. A small gap 25-30 $\mu \mathrm{m}$ directly opposite the ICM in the morning of day 5 or 6 .

- After drilling, incubation of the biopsy for four to enable blastocoel development and spontaneous herniation of trophectoderm cells

- Division of 3-10 trophectoderm cells through laser pulses

- Following biopsy, cells should be washed through multiple droplets prior to tubing. It is important to visually confirm that an intact cell has been placed into the lysis buffer. Cells should be collected into no more than $\sim 2.5 \mu$ l of buffer in 0.2 ml Flat Top PCR tubes figure 3.

A

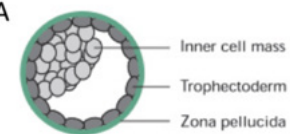

C

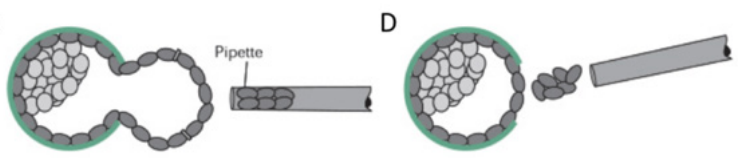

Figure 3: The main steps to perform trophectoderm biopsy.

Three main methods can be performed while doing the TE biopsy: the first start with an opening the zona pellucida at cleavage stage by laser-assisted drilling till the formation of an enlarged blastocyst on day 5. The reason behind drilling the Cleavage embryo zona is to obtain a faster biopsy on an expanded blastocyst and decrease the risk of unexpected collapse. Still, this method has a notable limitation; the embryo will be moved out of the incubators for two times for manipulation, and the substantial risk of having the inner cell mass herniating outside the zona. The second method is to apply assisted laser hatching for the TE, after the full blastocyst expansion. This approach needs a single embryo intervention, and the zona can be targeted in a range far from the inner cell mass, decreasing its association in the biopsy method. The last approach mixes the previous techniques in which it consists of penetrating the zona when the blastocyst is completely expanded and then waiting for the TE herniation [4,5] (Greco., et al. 2020).

There are several molecular techniques to perform PGT-A, as shown in the table below.

\begin{tabular}{|c|c|}
\hline $\begin{array}{l}\text { Molecular tech- } \\
\text { niques }\end{array}$ & Descriptions \\
\hline $\begin{array}{l}\text { comprehensive } \\
\text { chromosome } \\
\text { screening (CCS) } \\
\text { using array com- } \\
\text { parative genomic } \\
\text { hybridization } \\
\text { (aCGH) }\end{array}$ & $\begin{array}{l}\text { The method works through identifying al- } \\
\text { terations in the } 24 \text { chromosomes numbers } \\
\text { and rearrangements when analysing the } \\
\text { biopsied cells with a reference sample. The } \\
\text { sample is visualised through labelling with } \\
\text { fluorescent probes and hybridised to a } \\
\text { DNA microarray. The visualised probes can } \\
\text { indicate any loss or gain of chromosomes, } \\
\text { after detecting fluorescence and analyse } \\
\text { aneuploidy and chromosomal rearrange- } \\
\text { ments (Rodrigo., et al. 2014) }\end{array}$ \\
\hline $\begin{array}{l}\text { Single Nucleotide } \\
\text { Polymorphism } \\
\text { Array }\end{array}$ & $\begin{array}{l}\text { SNP technique, also known as molecular } \\
\text { karyotyping, which a sensitive method } \\
\text { applied to perform high-resolution DNA } \\
\text { genome copy number analysis and to } \\
\text { identify segmental areas of homozygosity, } \\
\text { known as regions of copy neutral loss of } \\
\text { heterozygosity. SNP results are analysed } \\
\text { through the maternal and paternal origin } \\
\text { to assess the ploidy status (Noronha, Rohr } \\
\text { and Chauffaille, 2015). }\end{array}$ \\
\hline Real-time PCR & $\begin{array}{l}\text { Real time PCR is a laboratory-based } \\
\text { method, which is widely applied to allow } \\
\text { dependable detection and quantification of } \\
\text { a targeted DNA genetics materials. }\end{array}$ \\
\hline $\begin{array}{l}\text { Next generation } \\
\text { sequencing }\end{array}$ & $\begin{array}{l}\text { NGS is a new technique that permits huge- } \\
\text { ly parallel sequencing, through cutting the } \\
\text { DNA into small pieces and binding them to } \\
\text { adapters to generate sequencing library. } \\
\text { NGS has multiple clinical applications from } \\
\text { genetics to infectious disease to ART in } \\
\text { clinics and laboratories (Hsiao, 2019). }\end{array}$ \\
\hline
\end{tabular}

Table 1

\section{PGT-A goals/indication}

PGT-A is applied to enhance and increase the rate of a healthy pregnancy and livebirth of genetically normal live births in couples 
undergoing ART. Furthermore, PGT-A procedure is mostly performed for couples with a history of recurrent miscarriages, recurrent implantation failure advanced maternal age, and male infertility factors (Cárdenas-Nieto., et al. 2020). Moreover, PGT-A aims to enhance the processes of a section of embryos for transfer and hence reduce the time to live birth (Wilding., et al. 2019). On the other hand, some studies investigated how PGT-A can be cost-effective for couples attempting ART; for instance, a new study observed that IVF with PGT-A to be cost-effective for women over the age of 37 years, the study considered the exact cost estimations for all women $>37$ years old and assumed that they would have received a blastocyst from a single IVF cycle, therefore reducing the most deficient diagnosis patients (Orvieto, 2018). Besides, several studies point out the importance of PGT-A in increasing the utilization of single embryo transfer (SET). Several advocate PGT-A to improve the employment of SET in patients having IVF treatment (Penzias. et al. 2018). As an example, Ubaldi., et al. 2015 research examined IVF success before and after modifying the clinic protocol created to reduce the number of transferred embryos in patients older than 35 years. SET was suggested for patients with less than two implantation failures. There were no significant differences in clinical pregnancy rates per transfer in both pre- and post- clinical treatment protocols. Still, there was considerable growth in livebirth rates per embryo transfer cycle for the SET/PGT-A recipients. Nonetheless, when comparing live-birth rates per cycle, there was no significant difference between groups (20.9\% without PGT-A vs 24.4\% with PGT-A) (Ubaldi., et al. 2015). Moreover PGT-A is highly recommended for advanced maternal age, since the increase in maternal age and induce oocytes and embryo chromosomal abnormalities. Several studies indicate that chromosomal irregularities increase gradually from age 31 through age 43, with aneuploidy rate of approximately 85\%. For example, in Munné., et al. 2005 study, they founded that before PGD, recurrent miscarriage patients had lost $87 \%$ of their pregnancies, while after PGT-A tests, they only lost $16.7 \%$ pregnancies. This difference was mainly due to decrease in pregnancy loss in the $>$ or $=35$-years age (Munné., et al. 2005). Moreover, Furthermore, Rubio., et al,2017 study performed a multi-centre randomized two-arms trial: the first group consist of woman (38-41 years) undergoing 24-chromosome screening PGTA on day-3 embryos, with a blastocyst transfer. The second group is the control group without PGT-A chromosome screening. In PGT-A group, $78.6 \%$ of embryos were aneuploid, a total of 37 pregnancies were achieved successfully with only one clinical miscarriage, with a delivery rate of $52.9 \%$ per transfer. In contrast, a total of 41 pregnancies were obtained in the control group, however, there where 16 miscarriages, and a delivery rate of $24.2 \%$ per transfer. The authors concluded that PGT-A screening is greater compared with controls, not only in clinical result at the first embryo transfer but likewise in considerably declining miscarriage rates and short- ening the time to pregnancy (Rubio., et al. 2017) [6-8]. Likewise, a single-center observational-cohort study included a total of 2538 couples in the control group and 308 patients were into (PGT-A) group. The study results showed the PGT-A group had better clinical outcomes (live-birth rate per transferred embryo, LBR $40.3 \%$ vs $11.0 \%$ ) and reduced multiple pregnancy rate (MPR, $0 \%$ vs $11.1 \%$ ) and pregnancy loss (PL, 3.6\% vs $22.6 \%$ ) in in advanced maternal age patients (Sacchi., et al. 2019). Most notably, PGT-A can help in the treatment of patients with unexplained recurrent recurrent pregnancy loss, once considering that embryo aneuploidies can be the reason of miscarriages. Numerous reports applying genetic testing in patients with this suggestion have displayed a reduction in miscarriage rate. Hodez-Wertz., et al. reported that, in a total of 2282 embryos examined, 60\% were aneuploid. Euploid embryo transfers performed were 181, with an implantation rate of $45 \%$ and ongoing pregnancy rate of $92 \%$. The miscarriage rate was only $6.9 \%$, as compared with the expected rate of $33.5 \%$ in a recurrent pregnancy loss control and $23.7 \%$ in infertile control population (Greco., et al. 2020) [9-11]. In addition to maternal factors, there are some aneuploidies which might arise from the spermatozoa. Males with abnormal karyotype and Y chromosome deletions manage to produce spermatozoa with an unbalanced chromosome. Other various factors, such as varicocele, chemotherapy, age, and lifestyle, can further have a negative impact on meiotic divisions through spermatogenesis (Greco., et al. 2020) [12]. Several studies have confirmed that PGT-A should be used in ICSI cycles with severe male factor (SMF), including azoospermia (obstructive and non-obstructive), severe oligoastenoteratozoospermia, Klinefelter syndrome (KS), Y-chromosome microdeletion, and even in men with a low results semen analysis. Magli., et al. illustrated that SMF could give to a greater rate of aneuploid blastocysts (55\% aneuploidy rate with normozoospermic, $62 \%$ with oligozoospermia, and 69\% with nonobstructive azoospermia (Magli., et al. 2009) [13]. Moreover, in another reported study, 72 male were recruited, of which 52 male had infertility and 20 male where assigned into the control group. The rate of abnormal sperm FISH examination was significantly higher in the patients' group (55.8\% vs $15.0 \%$ for controls). Asthenozoospermia, oligozoospermia and teratozoospermia were significantly correlated with the detection of abnormal FISH examination. Teratospermia was significantly correlated with increased aneuploidy rate for chromosome $17(\mathrm{p}=.005)$, chromosome $X(p=.05)$ and $Y(p=.03)$ (Petousis., et al. 2017). When to perform PGT-A? With the big conflict around if PGT-A is has a real influence on pregnancy rate and live birth. As a result, it is possible that patients are getting conflicting suggestions about the use or disuse of this procedure. Besides, there are no enough data regarding the benefits of PGT-A (Quinn., et al. 2017) [14-16], The study made to assess why patient undergoes PGT-A, founded that three-quarters of all patients who chose PGT-A meant they did 
so to either have a healthy child or decrease the risk of congenital disabilities. Besides, nearly one in six individuals thinking to undergo PGT-A is mostly to decrease miscarriage risk. Early pregnancy loss is mainly due to aneuploidy, providing biologic plausibility for the use of PGT-A amongst patients seeking to avoid miscarriage. Nevertheless, data have not yet confirmed a relationship between its use and this outcome (Figure 4) (Quinn., et al. 2017).

Why patients decide to
undergo PGT-A ?
- To have healthy baby
- Reduce miscarriage
- Reduce birth defects
- Reduce time to
pregrency

Why patients decide not
to undergo PGT-A ?
- Cost
- Reduce harm from
biopsy
- Avoid no transfer
- Healthy baby

Figure 4: The figure above illustrate the main reasons behind choosing to undergo or refuse to perform PGT-A (Quinn., et al. 2017).

\section{PGT-A interpretation}

PGT-A for chromosome irregularities has been applied to enhance assisted reproductive technology results for almost 20 years. However, in some cases, the effectiveness of (PGT-A) may be decreased, since the source of the mistakes throughout PGT-A is low sensitivity or specificity of either the method or interpretation. Besides, the threshold value which classifies normal result from pathological is not completely formalized for PGT-A. The decision on the status of the sample is taken by the interpreter (the expert) [18,19], (Musatova and Pomerantseva, 2019).

The below graph illustrates the process of results interoperation according to ESHREA.

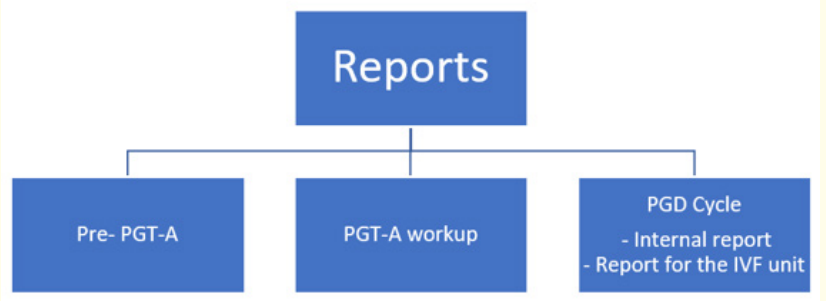

Figure 5: The figure above illustrate the categorising of PGT-A results reporting (Fiorentino, 2010).

No pre-implementation molecular diagnostics methods can give a $100 \%$ sensitivity (no false negatives), specificity (no false positives), and accuracy, this is due to that embryos are extremely dynamic structures, where embryonic cells divide very dynamically, thus genetic errors in the testing can occur. In a simple way results interpretation can be categorized as recommended to be transferred, not recommended to be transferred, or 'recommended upon signing the consent form.

\section{Non-invasive PGT-A}

PGT-A has been widely used to improve implantation and pregnancy rates. Still, there are some concerns regarding PGT-A, such as that it is not yet applied as a stander screening test for all IVF patients. In addition, the process of PGT-A requires an embryo biopsy procedure, that is considered to be invasive and may have a possibly harmful influence on the embryo developmental potential (Yeung., et al. 2019) [20,21]. Even if the trophectoderm (TE) biopsy is performed, likely, the genetic composition of TE may not represent that of the inner cell mass. Thus, a non-invasive technique that can estimate the embryo ploidy state can be an ideal solution for embryo genetics screening tests (Yeung., et al. 2019) [22,23]. Noninvasive preimplantation genetic testing for aneuploidy (niPGT-A) was firstly developed in 2016, to prevent the embryo development distracting and removing extra cells from the embryo, through analysing DNA leaked from human blastocysts into the culture medium. In one study, where they used niPGT-A method, to perform chromosome screening on IVF embryos from seven couples with balanced translocation, azoospermia, and recurrent pregnancy loss. The screening results revealed that six of them completed successful clinical pregnancies and healthy live births. This confirms that niPGT-A technique prevents the need for embryo biopsy and, consequently, this will considerably increase the safety of its use (Xu., et al. 2016) [24-26]. Despite all the advantages of inPGT-A during the past years, it still has some downfaults due to the occurrence of a percentage of false-positive diagnoses in the blastocyst biopsy of the trophoblast, particularly in conditions of embryonic mosaicism. As an improvement solution, a validation program is necessary before obtaining free-DNA in a spent culture medium, to prevent DNA-contamination. For instance, some software can use artificial intelligence to identify suspected of contamination in DNA cases. Besides, the procedure for collecting free-DNA need to be strictly regulated for each laboratory. This involves special pipettes, culture plates suitable for reduced volumes of culture medium, and the measurement of the use of a sequential or continuous culture system, depending on each laboratory's routine [27] (Franco Júnior, Dieamant and Oliveira, 2020).

\section{Conclusion}

In assisted reproduction, high-quality ovum is the basic and primary requirement for successful IVF [29]. Increasing the number of retrieved oocytes is an effective way to improve the number of 
transplantable or even high-quality embryos. Repeated flushing and extended time required for oocyte recovery during the process of oocyte retrieval, significantly reduced oocyte and embryo development potential.

\section{Bibliography}

1. Agarwal A., et al. "A unique view on male infertility around the globe". Reproductive Biology and Endocrinology 13 (2015): 37.

2. Boada M and Veiga A. "Embryo Biopsy". Barcelona, Spain: INSTITUT UNIVERSITARI DEXEUS (2010).

3. Cárdenas-Nieto D., et al. "Analysis of a Preimplantation Genetic Test for Aneuploidies in Embryos from Colombian Couples: A Report of Cases". Journal of Reproduction and Infertility 21.1 (2020): 17-33.

4. Fiorentino F. "Reporting Of Results". Roma: ESHRE (2010).

5. Fragouli E., et al. "The cytogenetic constitution of human blastocysts: insights from comprehensive chromosome screening strategies". Human Reproduction Update 25 (2018): 15-33.

6. Franco Júnior J., et al. "Noninvasive preimplantation genetic testing for aneuploidies (niPGT-A) and the principle of primum non nocere". JBRA Assisted Reproduction 24.4 (2020): 391-393.

7. Greco E., et al. "Preimplantation Genetic Testing: Where We Are Today". International Journal of Molecular Sciences 21.12 (2020): 4381.

8. Harper J., et al. "Recent developments in genetics and medically-assisted reproduction: from research to clinical applications†‡". Human Reproduction Open 3 (2017).

9. Hsiao S. "Sources of error in molecular diagnostic analyses". Accurate Results in the Clinical Laboratory (2019): 337-347.

10. Kokkali G., et al. "ESHRE PGT Consortium and SIG Embryology good practice recommendations for polar body and embryo biopsy for PGT†". Human Reproduction Open 3 (2020).

11. Lal A., et al. "Embryo Biopsy Can Offer More Information Than Just Ploidy Status". Frontiers in Cell and Developmental Biology 8 (2020): 78.

12. Magli M., et al. "Paternal contribution to aneuploidy in preimplantation embryos". Reproductive BioMedicine Online 18.4 (2009): 536-542.

13. Munné S., et al. "Preimplantation genetic diagnosis reduces pregnancy loss in women aged 35 years and older with a history of recurrent miscarriages". Fertility and Sterility 84.2 (2005): 331-335.

14. Musatova E and Pomerantseva E. "39. Clinical interpretation of non-standard pgt-a results". Reproductive BioMedicine 39 (2019): e50-e51.
15. Noronha T., et al. "Identifying the similarities and differences between single nucleotide polymorphism array (SNPa) analysis and karyotyping in acute myeloid leukemia and myelodysplastic syndromes". Revista Brasileira de Hematologia e Hemoterapia 37.1 (2015): 48-54.

16. Orvieto R. "The cost of a euploid embryo identified from preimplantation genetic testing for aneuploidy (PGT-A)". Journal of Assisted Reproduction and Genetics 35.11 (2018): 20772077.

17. Penzias A., et al. "The use of preimplantation genetic testing for aneuploidy (PGT-A): a committee opinion". Fertility and Sterility 109.3 (2018): 429-436.

18. Petousis S., et al. "Fluorescence in situ hybridisation sperm examination is significantly impaired in all categories of male infertility". Andrologia 50.2 (2017): e12847.

19. Quinn M M., et al. "Decision-making surrounding the use of preimplantation genetic testing for aneuploidy reveals misunderstanding regarding its benefit". Journal of Assisted Reproduction and Genetics 35.12 (2018): 2155-2159.

20. Rodrigo L., et al. "New Tools for Embryo Selection: Comprehensive Chromosome Screening by Array Comparative Genomic Hybridization". BioMed Research International (2014): $1-9$.

21. Rubio C., et al. "In vitro fertilization with preimplantation genetic diagnosis for aneuploidies in advanced maternal age: a randomized, controlled study". Fertility and Sterility 107.5 (2017): 1122-1129.

22. Sacchi L., et al. "Preimplantation Genetic Testing for Aneuploidy Improves Clinical, Gestational, and Neonatal Outcomes in Advanced Maternal Age Patients Without Compromising $\mathrm{Cu}$ mulative Live-Birth Rate". Journal of Assisted Reproduction and Genetics 36.12 (2019) 2493-2504.

23. Ubaldi F., et al. "Reduction of multiple pregnancies in the advanced maternal age population after implementation of an elective single embryo transfer policy coupled with enhanced embryo selection: pre- and post-intervention study". Human Reproduction 30.9 (2015): 2097-2106.

24. Viotti M. "Preimplantation Genetic Testing for Chromosomal Abnormalities: Aneuploidy, Mosaicism, and Structural Rearrangements". Genes 11.6 (2020): 602

25. Wilding M., et al. "Thaw, biopsy and refreeze strategy for PGTA on previously cryopreserved embryos". Facts, Views and Vision in ObGyn 11.3 (2019): 223-227.

26. Xu J., et al. "Noninvasive chromosome screening of human embryos by genome sequencing of embryo culture medium for in vitro fertilization". Proceedings of the National Academy of Sciences 113.42 (2016): 11907-11912. 
27. Yeung Q., et al. "A prospective study of non-invasive preimplantation genetic testing for aneuploidies (NiPGT-A) using nextgeneration sequencing (NGS) on spent culture media (SCM)". Journal of Assisted Reproduction and Genetics 36.8 (2019): 1609-1621.

\section{Assets from publication with us}

- Prompt Acknowledgement after receiving the article

- Thorough Double blinded peer review

- Rapid Publication

- Issue of Publication Certificate

- High visibility of your Published work

Website: www.actascientific.com/

Submit Article: www.actascientific.com/submission.php

Email us: editor@actascientific.com

Contact us: +919182824667 